\title{
Adaptive Watermarking based on Tree Structure based using 3-4 Level DWT and DCT
}

\author{
Savneet kaur \\ Indo Global Group of Colleges
}

\author{
Kumud sachdeva \\ Assistant professor \\ Indo Global Group of Colleges
}

\begin{abstract}
An adaptive watermarking scheme provides more contribution related to DWT for image content. In this paper an adaptive watermarkingschemeusing tree structure is proposed.This watermarking method makes use of a classificationprocedure for identifying various parts of the image which can be watermarkedby using the most suited modulation DWT. This classificationdependson a reference image which is derived from the image itself and aprediction of it necessarywhich has the property of being invariant change tothe watermark insertion process.In this paper the results on the basis of psnr, mse, entropy and embedding capability, mean ssim, just noticeable difference have been found. This algorithm is better for the psnr and embedding capability as compared to the previous algorithm. Proposed algorithm has improved successful watermarking image for future work with other compatible algorithm for the security purpose.
\end{abstract}

\section{Keywords}

DWT, Watermarking, Tree structure, DCT, Robustness, embedding capability, entropy, JND

\section{INTRODUCTION}

Digital watermarking is an important concept of the digital world. In watermarking process, form, image or text are impressed onto paper. In the recent years, the internet's growths has highlighted to the mechanisms to privacy in the digital world. This is applied for some information by embed within a digital media technology so that the inserted data becomes important part of this media. This technique serves for different number of purposes such as broadcast monitoring system, data authentication system, data indexing system. Watermarks have two categories for used: In the first category, the watermark consists as a transmission code and then decoding must used to recover the whole process transmitted information correctly. In the second category, the watermark consists as a verification code to identify. Digital watermarks consists the pieces information which added to digital data such as audio, video or still images that can be detected or extracted for assertion of the data. The watermark detector must simply to calculate the performance of a specific pattern in the latter system for used. Since the footprint of the verification watermarking scheme such as more number of pixels per watermark code bit is typically higher for apply. This case gives higher robustness and better performance as compared to the subliminal channel case. In watermarking schemes, the watermark message is embedded to the host signal in different purpose such asadditively or multiplicatively. To restore exactly the original image from its watermarked version by removing the watermark extraction done by using this method. It is possible to update the watermark content as for example security attributes i.e one digital signature or some authenticity codes at any time without adding new image distortions. As the introduction of the concept of adaptive watermarking in the Barton patent there are several schemes. Most methods use Expansion Embedding modulation, DWT modulation ormany more recentlyuse their combination.

A digital watermarking technique provides a solution such as longstanding problems faced with copyrighting digital data. Among these solutions may use in most recent schemes for Expansion embedding modulation, now days, DWT modulation is more recent combination in the watermarking process. The main concept with these modulations is to avoid underflows as well as overflows.With help of adding watermark signal to the image caution process must be taken to avoid gray level value such as underflows (negative) and overflows which isgreater than a bit depth image for watermarked image at the time of minimizing image distortion.

\section{WATERMARKING PROCESS}

Digital Watermarking software searches for noise in digital media technology and replaces this noise with useful information. The watermarking process includes three steps for this process such as watermark Insertion, Extraction and Detection. A digital media file is a type of list which contains a large list of 0 's and 1's. The watermarking software determines which of these 0's and 1's correspond to redundant or irrelevant details.

\section{REQUIREMENTS OF WATER MARKING}

Theinvisible watermarked document should satisfy several criteria used to be effectivein the protection of the ownership on demand of intellectual property.

- The watermark must be difficult or itshould be impossible to remove at least without applying the visibly of degrading the original image,

- The watermark must need to preserveoriginal image modifications that are common property in typical image-processing applications like scaling, color re-quantization, dithering, cropping, and image compression.

- Awatermark must imperceptible such as it should not beaffecting the experience of viewing the image.

- For some invisible type of watermarking applications, watermarks should be fast detectable by the proper authorities even if imperceptible to the average observer. Such decidability without requiring the original and unwatermarked image would be necessary for efficient recovery of property and subsequent prosecution. 
Embedding stage: One of the most important features that make the recognition of images possible by humans is color. Color contains the property that depends on the reflection of light to the eye and the processing of that information in the brain. The color is used every day to tell the difference between the objects, places and the time of day. Usually colors are defined in three dimensional color spaces usually colors are defined in three dimensional color spaces. These could be RGB i.eRed, Green, and Blue, HSV i.e Hue, Saturation, and Value and HSB (Hue, Saturation and Brightness).HUE and HSB are dependent on the human perception of hue, saturation, and brightness. Color represents the distribution of colors within the entire image. This representation includes the amount of each color but not the locations of colors.

\section{WATERMARKING ALGORITHM}

In this approach, a block based DCT algorithm is developed to embed the information to binary watermark for the color host image. Since some high frequency components may be discarded in image processing operation such as JPEG compression, the very low frequency components of the color host image will be works for the watermark embedding.

The Embedding Algorithm: In embedding technique has presented for the color images, these color image are decomposed into three components $\mathrm{R}, \mathrm{G}$ and $\mathrm{B}$. Watermark information will be embedded in the $\mathrm{G}$ plane using equation 1 to produce G'. Assume that $f(i, j)$ represents the pixel of the component of the RGB representation of the color host image, $w(i, j)$ represents the binary pixel of the watermark.

$$
\begin{aligned}
& F_{k}(u, v)=\operatorname{DCT}\left\{f_{k}(i, j)\right\}, \\
& \text { If } \boldsymbol{w}(i, j)=1 \text { then }
\end{aligned}
$$

$$
F_{k}(x, y)=\left\{\begin{array}{ccc}
\Delta Q_{e}\left(\frac{F_{k}(x, y)}{\Delta}\right) & x, y \in H_{k} & 1 \leq k \leq N_{H B} \\
F_{k}(x, y) & x, y \notin H_{k} & 1 \leq k \leq N_{H B}
\end{array}\right\}
$$$$
\text { If } w(i, j)=0 \text { then }
$$

$$
F_{k}(x, y)=\left\{\begin{array}{ccc}
\Delta Q_{o}\left(\frac{F_{k}(x, y)}{\Delta}\right) & x, y \in H_{k} & 1 \leq k \leq N_{H B} \\
F_{k}(x, y) & x, y \notin H_{k} & 1 \leq k \leq N_{H B}
\end{array}\right\}
$$

Where Qe is the quantization to the nearest even number and Qo is the quantization to the nearest odd number $\Delta$ is a scaling quantity and it is also the quantization step used to quantize either to the even or odd number. The predefined coefficients in each $8 \times 8$ sub block are represented by NHB.

\section{TREE STRUCTURE BASED WATERMARK EMBEDDER}

The tree structure based watermark embedder is designed to embed the binary watermark bits into the selected bit planes of the selected DWT coefficients of the selected trees. In the proposed scheme, the tree structure based watermark embedder has three functions: forming the tree structure, selecting the trees and the DWT coefficients for the watermark embedding, and embedding the binary watermark bits into the selected bit planes of the selected coefficients. All these functions will be presented in the following three subsections.

- The Formation of the Tree Structure

- $\quad$ Selection Trees and DWT Coefficients

- $\quad$ The Watermark Embedding
Extracting stage: In a digital watermarking technique, it is not simple to carry the original image every time in order to detect the owner's information from the watermarked image. For this type of applications which is required different watermarks techniques for different copies so that it is easy topreferable and simple to utilize for some kind of watermark-independent algorithm for extraction process such as de-watermarking. It's give robustness against many attacks including rotation, low pass filtering, salt $\mathrm{n}$ paper noise addition and compression. For better activeness, watermark should be perceptually notvisible within host media statistically invisible to unauthorized removal and readily extracted by owner of image robust to accidental and intended signal distortion as filtering,compression,resampling,retouching, crapping etc. For a digital watermark to be effective for ownership, it must be robust, fragile from a document.It should provide the reliable original information toembed that should be removed by authorized users only.

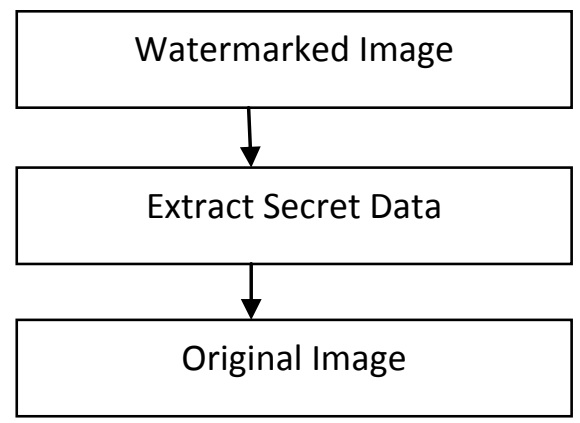

Fig: Extracting stage

The Extraction Algorithm:The embedded information w (i,j) can be extracted by performing $8 \times 8$ DCT transform for the watermarked host image and indicate the same coefficients of the host image that carries the 16 bits of the embedded watermarks using the same secret key in the initial scrambling operation.

$$
\begin{aligned}
& \text { If } Q\left(\frac{F_{k}(x, y)}{\Delta}\right) \text { is odd then } w(i, j)=0 \\
& \text { If } Q\left(\frac{F_{k}(x, y)}{\Delta}\right) \text { is even then } w(i, j)=1
\end{aligned}
$$

Here $\mathrm{Q}$ is rounded to the nearest integer and the scaling quantity is the same as the one used in the embedding process.

Robustness: The watermark should be robust such that it must be difficult to extracts. The watermark should be robust to different attacks. The robustness explains watermark can be reliably detected after performing some media operations.

\section{DISCRETE WAVELET TRANSFORM}

Discrete wavelet transform (DWT) is any wavelet transform which contains the discretely sampledwavelets. As compared with other wavelet transforms ,main advantage it has over Fourier transformsis precision of a measurement with respect to location in time.DWT contains decomposition of image into frequency channel of constant bandwidth. This causes the similarity of available decomposition at every level and DWT is implemented as multistage transformation. 


\section{DISCRETE COSINE TANSFORM}

A discrete cosine transform (DCT) describes a finite sequence of data points in terms of a sum of cosine functions oscillating at different frequencies. Discrete cosine transform are vitally usedto numerous applications in the field of science and engineering. From lossy compression of audioi.eMP3 and imagesi.eJPEGwhere small high-frequency components to spectral methods for the numerical solution of partial differential equationscan be discarded. The use of cosine as compared to sine functions is critical for compression as it turns out that some cosine functions are needed to approximate a typical signal whereas for differential equations the cosines express a particular choice of boundary conditions

\section{RESULTS AND DISCISSION}

PSNR - In table 1, PSNR measured to identify the quality of the watermarking image. It is measured on the basis of comparison of previous algorithm and the proposed algorithm. Proposed algorithm has shown improved psnr results for quality of watermarking.

\section{Comparison of PSNR between Previous and our algorithm

\begin{tabular}{|r|r|r|}
\hline & Previous Work Proposed Work \\
\hline PSNR & 43.9500 & 93.4798 \\
\hline
\end{tabular}

\section{Table1: Comparison of PSNR between previous and proposed algorithm}

In this the graphical representation is shown of the compared psnrresults follows as:

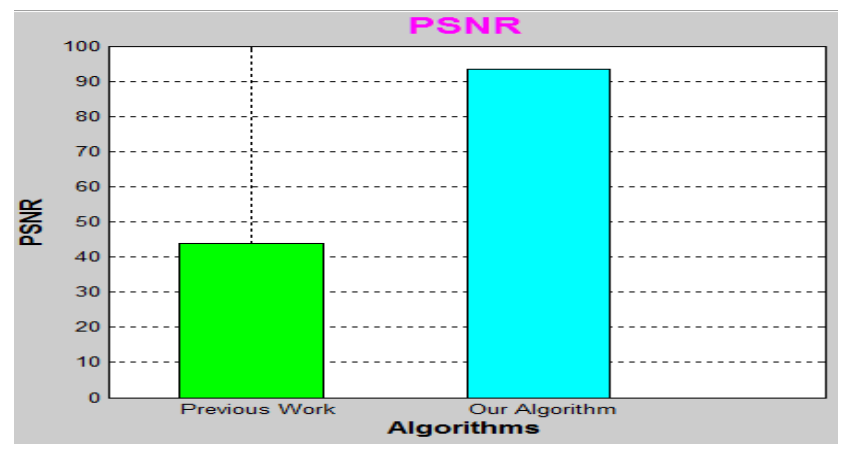

Figure 1, Comparison of PSNR graph

Embedding Capacity - In table 2, embedding capacity of the watermarking images is compared on the basis of previous algorithm and the proposed algorithm. Proposed method has given the more embedding capacity as compare to the previous algorithm for hide the information.

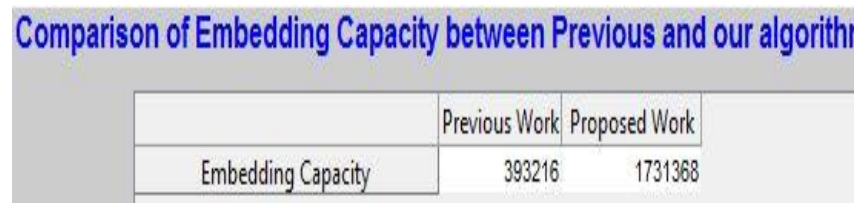

Table 2, Comparison of Embedding Capacity
In figure 2, the graphical representation is shown of the compared embedding capacity results follows as:

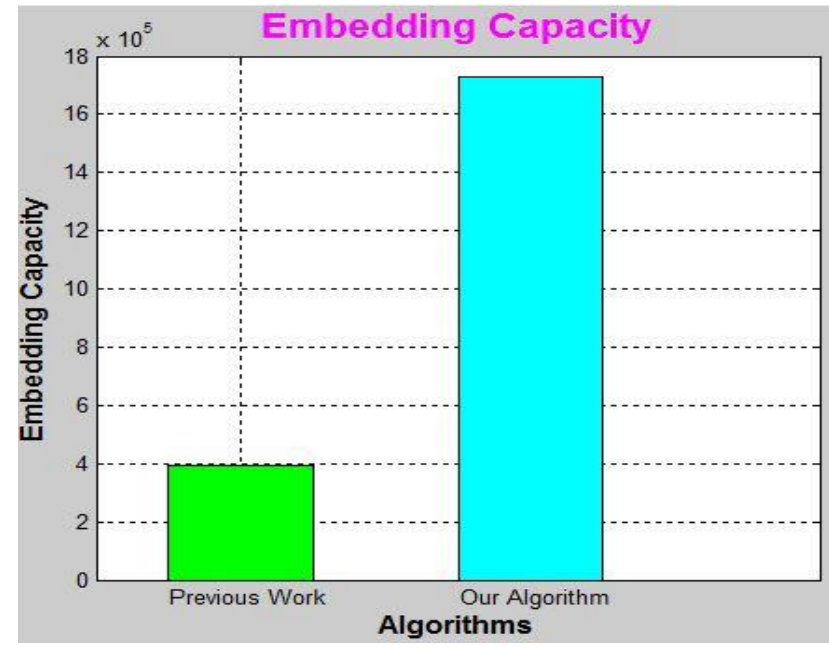

Figure 2, Comparison of Embedding Capacity graph of previous algorithm and proposed algorithm

Entropy - In figure3, the entropy is calculated for the watermarking image for proposed algorithm. It should be less for better watermarking used image.Proposed algorithm has improved the entropy upto 7.0817 follows as:

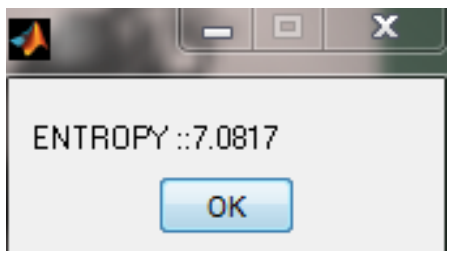

Figure 3, Entropy value of proposed algorithm

MSE - In figure 4,the mse value is calculated for the watermarking image to minimize the error. Proposed algorithm has been reduced the mseupto 0.1571 follows as:

\begin{tabular}{|r|r|}
\hline \multicolumn{2}{|c|}{ MSE of our algorithm } \\
\hline & Proposed Work \\
\hline MSE & 0.1571 \\
\hline
\end{tabular}

Figure 4, Comparison of Embedding Capacity graph

Just Noticeable Difference-Just Noticeable Difference is used to removes the disparities for the better quality. In table 5 , the just noticeable difference is found between the previous method and the proposed method. Proposed method has given the better resultsup to 0.3000 as compare to the previous method follows as:

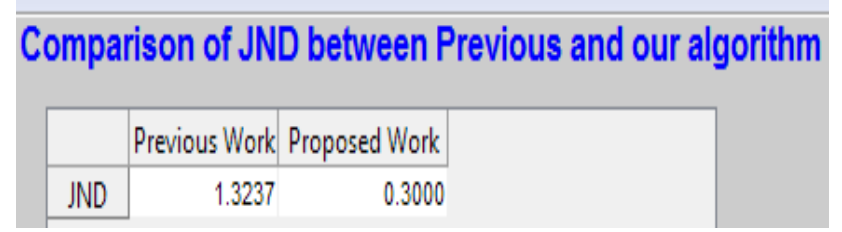

Table 5, Comparison of JND

In figure 5 , the comparison graph is shown of table 5 for the better visualization. 


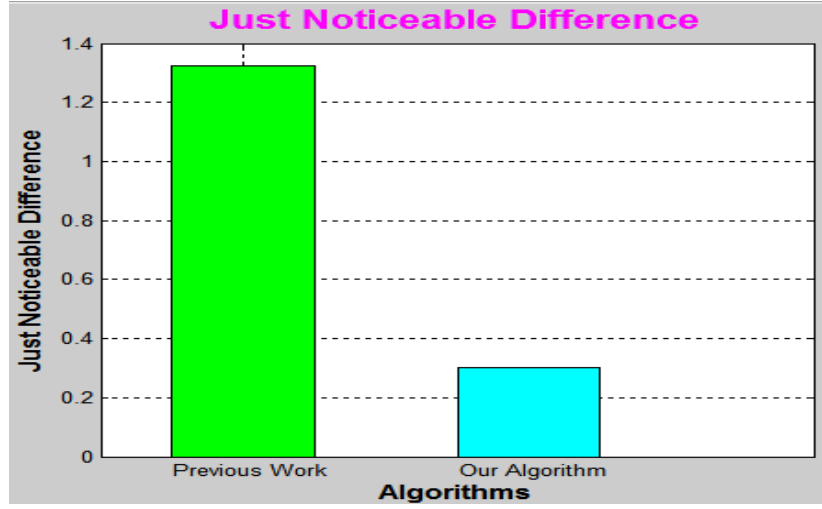

Figure 5, Comparison of JND of previous method and the proposed method

Mean SSIM - Mean SSIM is used to improve the limitations of the psnr and mse for better accuracy. In figure 6, shows the comparison graph of mean SSIM between previous method and the proposed method.

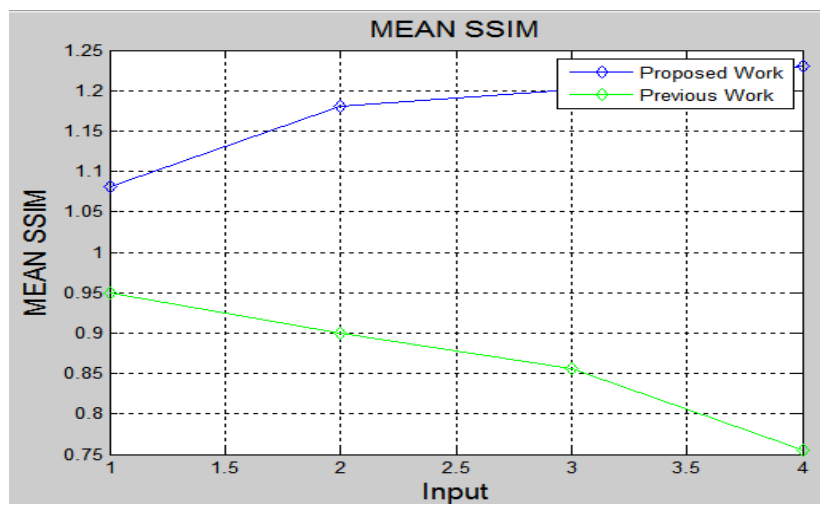

Figure 6, Comparison of Mean SSIM using previous method and the proposed method

\section{CONCLUSION AND FUTURE SCOPE}

In this paperbetter results have been found on the basis of proposed methodology of watermarking algorithm, proposed primary goal is on adaptive watermarking schemethat explains identifying each part of the watermarked image. In this watermarking scheme, hidden information of quality image tree structure is improved which contains quadtree decompositionas well as watermarking embedding algorithm tokeep the balance between watermarks' imperceptibility and itsrobustness. It's concluded from obtained results that asuitable domain of entropy and embedding capacity is improved.For further more improvement,Force field transform may be applied in future improvement with some watermarking scheme such as copyright infringement hindering in the court of law.

\section{REFERENCES}

[1] G. Coatrieux and Wei Pan," Reversible Watermarking Based on Invariant Image Classification and Dynamic Histogram Shifting", IEEE Transactions on information Forensics \& Security,vol 8,Issue 1, January 2013.

[2] Mr.Kamlesh.C.Badhe, andProf.Jagruti.R.Panchal,'Imple mentation of Audio Watermarking Using Wavelet Families", International Journal of Innovative Science, Engineering \& Technology, Vol 1, Issue 4, June 2014.
[3] Seema and Sheetal Sharma ," DWT-SVD Based Efficient Image Watermarking Algorithm to Achieve High Robustness and Perceptual Quality “, International Journal of Advanced Research in Computer Science and Software Engineering, Vol 2, Issue 4, April 2012.

[4] L. Luo, Z. Chen, M. Chen, X. Zeng, and Z. Xiong, "Reversible image watermarking using interpolation technique," IEEE Trans. Inf. ForensicsSecurity, vol. 5, no. 1, pp. 187-193, Mar. 2010.

[5] D. Coltuc, "Improved embedding for prediction-based reversible watermarking," IEEE Trans. Inf. Forensics Security, vol. 6, no. 3, pp. 873-882, Sep. 2011.

[6] C. C. Lin, W. L. Tai, and C. C. Chang, "Multilevel reversible data hiding based on histogram modification of difference images," PatternRecognition., vol. 41, pp. 3582-3591, 2008.

[7] C. H.Yang andM.H.Tsai, "Improving histogram-based reversible data hiding by interleaving predictions," IET Image Process., vol. 4, no. 4, pp. 223-234, Aug. 2010.

[8] P. Tsai, Y. C. Hu, and H. L. Yeh, "Reversible image hiding scheme using predictive coding and histogram shifting," Signal Process., vol. 89, pp. 1129-1143, 2009.

[9] D. Coltuc and J.-M. Chassery, "Distortion-free robust watermarking: A case study," in Proc. Security, Steganography, and Watermarking ofMultimedia Contents IX, San Jose, CA, 2007, p. 65051N-8.

[10] W. Pan, G. Coatrieux, N. Cuppens, F. Cuppens, and C. Roux, "An additive and lossless watermarking method based on invariant image approximation and Haar wavelet transform," in Proc. IEEE EMBC Conf., Buenos Aires, Argentina, 2010, pp. 4740-4743.

[11] V. Sachnev, H. J. Kim, J. Nam, S. Suresh, and Y.-Q. Shi, "Reversible watermarking algorithm using sorting and prediction," IEEE Trans.Circuit Syst. Video Technol., vol. 19, no. 7, pp. 989-999, Jul. 2009.

[12] H. J. Hwang, H. J. Kim, V. Sachnev, and S. H. Joo, "Reversible watermarking method using optimal histogram pair shifting based on prediction and sorting," KSII, Trans. Internet Inform. Syst., vol. 4, no. 4, pp. 655-670, Aug. 2010.

[13] H. M. Chao, C. M. Hsu, and S. G. Miaou, "A datahiding technique with authentication, integration, and confidentiality for electronic patient records," IEEE Trans. Inf. Technol. Biomed., vol. 6, no. 1, pp. 46-53, Mar. 2002

[14] Rakesh Ahuja S.S. Bedi Himanshu Agarwal, "A Survey of Digital Watermarking Scheme", MIT International Journal of Computer Science \& Information Technology, vol. 2, issue 1, pp. 52-59, Jan. 2012.

[15] P.Ramana Reddy, Dr. Munaga.V.N.K.prasad, Dr D. Sreenivasa Rao, - Robust Digital Watermarking of Images using Wavelets.l International Journal of Computer and Electrical Engineering, Vol. 1, No. 2, June 2009. 